\title{
Glycosphingolipids-function and useage.
}

\section{Clifford Lingwood*}

Depts. Laboratory Medicine \& Pathobiology and Biochemistry, University of Toronto, Canada

Accepted on 25 November, 2017

\section{Editorial}

Our research concerns the synthesis and function of glycosphingolipids (GSLs) in eukaryotic cells. Despite extensive structural characterization of these sugar-lipid conjugates, defining their function is difficult due to poor aqueous solubility. The fact that no one gene encodes their synthesis and membrane GSL organization within micro domains or 'rafts', is dependent on both their carbohydrate and lipid composition, further complicate functional studies. Membrane GSLs often provide the interface between pathogenic microbes and/or their products and host cells, making GSL-based therapeutics a possible mode of infection control. In turn, microbial GSL-binding ligands provide probes to study GSL function. The GSL lipid moiety and membrane environment regulate GSL receptor function and likely, biosynthesis. We have made species which mimic some of these features. Understanding the basis of this 'aglycone' effect is a central focus. We have found cholesterol masking of GSL carbohydrate via conformational restriction of the GSL carbohydrate is an important feature of GSL receptor function. A H-Bond network results in the carbohydrate adopting a membrane parallel rather than membrane perpendicular conformation. This renders the carbohydrate less available for trans-ligand binding-e.g. antibodies (invisible GSLs). The carbohydrate now forms an 'umbrella' over the steroid $\mathrm{OH}$, restricting the access of cholesterol binding ligands also. Cholesterol masking of GSLs is particularly important in tumour cells.

The higher tumour cell cholesterol content results in the masking of the majority of tumour associated GSLs from antibody recognition and thereby plays an important role in tumour evasion of immune surveillance. Strategies to deplete tumour cholesterol should reverse this masking. Several MAbs in clinical antineoplastic use, target GSLs and tumour biopsy binding is increased by cholesterol depletion.

We have worked extensively on the E.coli elaborated AB5 verotoxin-VT (aka Shiga toxin-Stx) and showed the B subunit binds to the GSL, globotriaosyl ceramide (Gb3). Gb3 is upregulated in many human tumours ovarian, breast, renal, gastric, pancreatic, brain, colon, lymphoma, and their neovasculature. Thus VT has both anti-tumour and anti angiogenic potential. We showed VT efficacy in a brain tumour xenograft model and this has been verified with many other tumour models. The ABC cassette transporter and multiple drug resistance pump, MDR1 (and some homologues) plays an important role in the trans membrane supply of precursor GSL for the synthesis of Gb3 (and other GSLs). This means that MDR1 overexpression in drug resistant tumours can lead to increased Gb3 content and thereby increased VT sensitivity.

Following ligand binding, cell surface GSLs undergo internalization and a process of retrograde transport from endosomes to the trans Golgi network to the ER. GSL binding bacterial toxins e.g. VT, cholera toxin use this pathway to target the ER where the A subunit is translocated to the cytosol via hijacking the ER retro translocon used for ER associated degradation (ERAD) of partially misfolded nascent proteins. We have used this property as a means to temporarily competitively block ERAD in the genetic diseases in which ERAD eliminates the mutant protein and induce/exacerbate disease symptoms (e.g. F508delta CFTR cystic fibrosis, Gaucher disease). This GSL dependent blockade partially reverses disease symptoms in cell culture and animal disease models, and provides a potential therapy for many misfolded protein based genetic diseases. ERAD blockade is also an antineoplastic stratagem. The HIV adhesion, gp120 also binds GSLs, including Gb3. We identified Gb3 as a host resistance factor against HIV and are using this to design novel inhibitors of HIV infection.

\section{*Correspondance to}

\section{Clifford Lingwood}

Prof. Depts. Laboratory Medicine \& Pathobiology and Biochemistry,

University of Toronto, Canada

E-mail: cling@sickkids.ca 